\title{
Monitoria acadêmica na percepção dos estudantes de enfermagem
}

Academic monitoring in the perception of students nursing

Tutoría académica desde el punto de vista de los estudiantes de enfermería

\section{Cinthya das Neves Burgos ${ }^{1}$, Crysthianne Cônsolo de Almeida Baricatii ${ }^{I I}$, Júlia Trevisan Martins ${ }^{I I I}$, Alessandro Rolim Scholze ${ }^{\mathrm{IV}}$, Maria José Quina Galdino ${ }^{\mathrm{V}}$ Marcia Eiko Karino ${ }^{\mathrm{VI}}$}

\begin{abstract}
Resumo: Objetivo: conhecer a percepção dos estudantes de enfermagem em relação à prática da monitoria acadêmica realizada em um laboratório de simulação de técnicas de enfermagem. Método: estudo qualitativo desenvolvido com 18 monitores, entre os meses de novembro e dezembro de 2017, por meio de entrevista semiestruturada. Os dados foram analisados pela técnica de análise de conteúdo. Resultados: a monitoria foi indicada pelos participantes como um despertar para docência, troca de experiência entre os mesmos, melhora nas relações interpessoais, momento de relembrar o que aprendeu na teoria, incentivo para estudar e fortalecimento da relação discente e docente. Considerações finais: a monitoria se constituiu como uma ferramenta importante para o desenvolvimento de habilidades teórico-práticas, que são importantes para o crescimento pessoal e profissional. Descritores: Ensino de enfermagem; Estudantes de enfermagem; Aprendizagem
\end{abstract}

Abstract: Aim: to know the perception of nursing students regarding the academic monitoring practice performed in a laboratory of nursing techniques simulation. Method: qualitative study, developed with 18 monitors, between November and December 2017, through semi-structured interview. The data was analyzed by the technique of content analysis. Results: the participants indicated monitoring as an awakening for teaching, exchange of experience among them, improvement in interpersonal relations, moment to remember what they had learned in theory, encouragement to study and strengthening of the student and teacher relationship. Final

\footnotetext{
I Enfermeira. Universidade Estadual de Londrina (UEL), Londrina, Paraná, Brasil, e-mail: cinthyaburgos@hotmail.com, ORCID: https://orcid.org/0000-0002-8161-5386.

II Enfermeira. Doutora em saúde coletiva. Universidade Estadual de Londrina (UEL), Londrina, Paraná, Brasil, e-mail: crysconsulo@hotmail.com, ORCID: https://orcid.org/0000-0001-6810-8008.

III Enfermeira. Doutora em enfermagem. Universidade Estadual de Londrina (UEL), Londrina, Paraná, Brasil, e-mail: jtmartins@uel.br, ORCID: https://orcid.org/0000-0001-6383-7981.

IV Enfermeiro. Mestre em enfermagem. Universidade Estadual do Norte do Paraná (UENP), Bandeirantes, Paraná, Brasil, e-mail: scholze@uenp.edu.br, ORCID: https://orcid.org/0000-0003-4045-3584.

v Enfermeira. Doutora em enfermagem. Universidade Estadual do Norte do Paraná (UENP), Bandeirantes, Paraná, Brasil, e-mail: mariagaldino@uenp.edu.br, ORCID: https://orcid.org/0000-0001-6709-3502.

VI Enfermeira. Doutora em ciências. Universidade Estadual de Londrina (UEL), Londrina, Paraná, Brasil, e-mail: marciak@uel.br, ORCID: https://orcid.org/0000-0002-6582-2801.
} 
considerations: monitoring has been an important tool for the development of theoretical-practical skills, which are important for personal and professional growth.

Descriptors: Education, nursing; Students, nursing; Learming

Resumen: Objetivo: conocer el punto de vista de los estudiantes de enfermería en relación a la práctica de la tutoría académica realizada en un laboratorio de simulación de técnicas de enfermería. Método: estudio cualitativo, desarrollado con 18 tutores, entre noviembre y diciembre de 2017, por medio de entrevista semiestructura. Los datos fueron analizados por análisis de contenido. Resultados: la tutoría fue indicada por los participantes como el momento del despertar para la enseñanza, del intercambio de experiencias, del mejoramiento de las relaciones interpersonales, del acordarse de la teoría, del estímulo para estudiar y del fortalecimiento de la relación entre estudiantes y profesores. Consideraciones finales: la tutoría es una herramienta importante para el desarrollo de habilidades teórico-prácticas, que son importantes para el crecimiento personal y profesional.

Descriptores: Educación en enfermería; Estudiantes de enfermería; Aprendizaje.

\section{Introdução}

Os cursos de graduação em enfermagem objetivam inserir e garantir ao estudante competência técnico-científica para o desenvolvimento de atividades assistenciais, gerenciais, de ensino e de pesquisa. ${ }^{1-2}$ Para ampliar as possibilidades de aprendizagem aos estudantes na execução dos procedimentos de enfermagem, tem-se as atividades acadêmicas complementares desenvolvidas por meio de monitorias nos laboratórios de simulação. ${ }^{3}$

Nesse sentido, as atividades de monitoria são consideradas um apoio pedagógico para os estudantes, utilizadas pelas disciplinas/módulos para que os mesmos possam praticar os procedimentos apresentados pelos docentes em sala de aula. Assim, as monitorias são conduzidas por estudantes, que foram capacitados para essa atividade, a fim de aprofundar conteúdos e/ou solucionar dificuldades de algum tema apresentados pelos estudantes. ${ }^{4}$ Portanto, a monitoria se configura como uma estratégia relevante para o processo de ensino/aprendizagem, visto que propicia segurança e precisão para os estudantes desenvolverem as competências durante as práticas clínicas, seja no ambiente hospitalar ou na atenção básica à saúde. ${ }^{5}$ 
Neste estudo, entende-se a monitoria acadêmica como uma forma de apoio ao ensino/aprendizagem, em que estudantes que já concluíram a disciplina contribuem no processo de apropriação do conhecimento de seus colegas, portanto, tem por finalidade auxiliar de maneira ativa no processo de aprendizagem, uma vez que esse monitor irá colaborar no aprimoramento dos estudantes quanto as técnicas de enfermagem e sua relação com a teoria. ${ }^{6} \mathrm{O}$ monitor é um ator do processo com quem os estudantes podem esclarecer suas dúvidas ou aprimorar seus conhecimentos e, por sua vez, melhorar o seu aprendizado. ${ }^{7}$

A legalização da monitoria no âmbito universitário foi instituída em 20 de dezembro de 1996, pela Lei de Diretrizes e Base da Educação Nacional, Lei n 9.394, que em seu artigo 84 indica que os alunos de graduação do ensino superior poderão ser inseridos em atividades de ensino e pesquisa pelas respectivas instituições, desenvolvendo funções de monitoria, desde que esteja em consonância como o projeto político pedagógico dos cursos. ${ }^{8}$

Autores $^{9}$ afirmam que é de fundamental importância estudos sobre a monitoria em curso de graduação em enfermagem, visto que as contribuições são relevantes para o processo de ensino/aprendizagem do estudante, ao propiciar a prática dos procedimentos e teoria que foram ensinados pelos professores em sala de aula e laboratório e, assim, contribui para um melhor aproveitamento do curso.

Diante do exposto, tem-se a seguinte questão: quais as percepções dos estudantes de enfermagem em relação à prática da monitoria acadêmica realizada em um laboratório de simulação de técnicas de enfermagem? Para responder a esse questionamento traçou-se como objetivo conhecer a percepção dos estudantes de enfermagem em relação à prática da monitoria acadêmica realizada em um laboratório de simulação de técnicas de enfermagem. 


\section{Método}

Trata-se de um estudo descritivo, com abordagem qualitativa, realizado com monitores de um curso de graduação em enfermagem de uma universidade pública do Paraná. O referido curso adota o currículo integrado, que tem como proposta pedagógica o ensino centrado no aluno, ou seja, ele é o protagonista do seu próprio aprendizado e compete ao docente ser o facilitador do ensino/aprendizado, o qual incentiva o raciocínio lógico partido do conhecimento prévio do estudante, isto é, de suas próprias experiências. ${ }^{2}$

O laboratório de enfermagem conta com um total de 18 monitores voluntários, que são responsáveis pelas monitorias dos módulos: Aspectos Morfofisiológicos na Enfermagem e Práticas Fundamentais na Enfermagem. Para selecionar os estudantes elegíveis neste estudo, adotou-se como critério de inclusão ter sido monitor no laboratório de enfermagem por, no mínimo, seis meses. A seleção dos monitores é realizada por edital elaborado pelos docentes do curso, o qual versa sobre os requisitos para a inscrição e avaliação dos candidatos. A seleção é composta por: prova de conhecimentos pertinentes ao módulo para qual está inscrito, análise de currículo e entrevista individual para estabelecer a ordem classificatória. São elegíveis todos os alunos que cursaram e estiveram aptos nos módulos descritos.

Os aprovados dentro do número de vagas são treinados pelos docentes antes de iniciar a monitoria. A vigência da monitoria é de um ano letivo e os monitores devem cumprir uma carga horária de 120 horas anuais, para tanto, é preciso cumprir 12 horas semanais, das quais oito são destinadas às atividades no laboratório de Enfermagem e quatro para o estudo dos módulos. Enfatiza-se, que a atividade dos monitores é exclusiva para fins de ensino, pois as monitorias para fins de pesquisa acontecem em outro módulo do curso em questão.

A coleta de dados foi realizada por intencionalidade, porém respeitou-se a convergência dos dados, assim, este estudo teve a participação de 13 monitores. Enfatiza-se que os monitores que participaram do estudo foram monitores do nos anos de 2016 e 2017. 
Para a coleta de dados, utilizou-se a entrevista individual semiestruturada, com a seguinte questão: Quais são suas percepções sobre ser monitor em um laboratório de enfermagem? A duração das entrevistas, em média, foi de 30 minutos. Estas foram audiogravadas e transcritas na íntegra. A coleta se deu entre os meses de novembro e dezembro de 2017.

Os dados foram analisados e interpretados seguindo a técnica de análise de conteúdo e teve as seguintes etapas: pré-análise; exploração do material e tratamento dos resultados, inferência e interpretação. Na pré-análise ocorreu à organização do material, isto é, realizou-se leitura flutuante das entrevistas para identificar as particularidades de cada entrevistado, que contribuiu para a elaboração das ideias iniciais. $\mathrm{Na}$ sequência, passou-se para a fase de exploração do material, ou seja, procedeu-se a execução da codificação das entrevistas letra e número, de maneira que os recortes que interessaram ao estudo foram agrupados em núcleos de sentido análogos e que originaram as categorias. Por fim, deu-se o tratamento dos resultados, no qual os resultados brutos foram tratados de maneira significativa e válida, havendo análise e uma discussão dessas falas provenientes das entrevistas. ${ }^{10}$

Os preceitos éticos foram respeitados, como o anonimato das informações, assinatura do Termo de Consentimento Livre e Esclarecido pelos pesquisados e a aprovação do Comitê de Ética, seguindo os preceitos éticos estabelecidos pela resolução no 466/2012. Assim, esta pesquisa teve o parecer CAAE no 75075417.5.0000.5231, sob o protocolo 2.313.845, em outubro de 2017. Para garantir o anonimato, os participantes foram identificados pela letra M, seguida de numeração de acordo com a ocorrência das entrevistas. 


\section{Resultados e discussão}

Dos 13 monitores, oito eram do sexo feminino e cinco do masculino, com faixa etária entre 21 e 46 anos, todos cursando o $3^{\circ}$ ano do curso de graduação em enfermagem. Por meio da exploração e organização das falas dos monitores, emergiram quatro categorias.

\section{O despertar para a docência}

Esta categoria abrange o significado e importância de ser monitor durante a graduação e, principalmente, o despertar para possibilidade de atuação profissional na docência. Conforme pode ser identificado nas seguintes falas:

Uma oportunidade que me fez ver outras opções de carreira, mesmo que não ministramos aulas teóricas, quando ensinávamos os procedimentos aos nossos colegas fez com que eu me interessasse pela docência, pois tínhamos que explicar a teoria para mostrar a prática. (M3)

Para mim, ser monitor significou uma iniciativa e um incentivo para a atividade docente, sendo essencial para despertar esse desejo. Aprendi que organizar, controlar e passar o conhecimento para outras pessoas, vi o quanto é difícil ser docente. (M6)

Pode-se observar que a monitoria desperta o desejo dos alunos em seguir a carreira de docente. Quando o estudante se torna um monitor, este passa a acompanhar as atividades acadêmicas desenvolvidas pelo professor responsável pela monitoria e, assim, conhece o contexto em que os educadores atuam o que favorece para a ampliação de seu conhecimento, abrindo caminhos e estratégias para o aumento do desenvolvimento didático-pedagógico desde a graduação e por sua vez despertando o interesse para seguirem a carreira de docente. ${ }^{2}$

Autores $^{11}$ afirmam ser de extrema importância a monitoria, pois propicia ao estudante desenvolver experiência e o despertar para ser docente, visto que, auxilia no processo de ensinoaprendizagem, bem como, ajuda a si próprio e aos colegas, no processo de aquisição de habilidades. 


\section{Troca de experiências e fortalecimento da relação estudante-estudante e estudante-docente}

Os estudantes consideraram que as atividades de monitoria constituíram momentos de troca de conhecimentos entre os próprios alunos, sendo uma oportunidade para fortalecer a relação entre os monitores e os docentes e, ainda, trouxe o aprendizado de trabalho em equipe. É o que mostra as seguintes falas:

Foi positivo para nós, pois aprendemos junto com os alunos que estávamos ensinando, houve troca de experiência e foi muito rico. Favoreceu a relação com os nossos colegas. Aprendemos tanto no quesito conhecimento, quanto no relacionamento com os alunos e professores. (M1)

Foi muito bom ter essa experiência e sentir a confiança que as professoras têm nos monitores. Fortaleceu minha relação com os professores e colegas. (M9)

$A$ assistência dos professores também foi ótima e percebi que a minha relação com eles ficou muito mais forte. (M12)

Gostei demais, aprendi a entender melhor os professores e fortaleceu demais o meu relacionamento não só com os professores responsáveis pela monitoria, mas com todos os docentes do curso e também com os meus colegas. (M13)

Constatou-se pelos depoimentos que os alunos consideram a experiência da monitoria como uma prática que proporcionou interação entre os colegas e os docentes, bem como se sentiram valorizados pela confiança que os docentes depositavam em suas atividades. Os monitores estão mais próximos dos seus colegas que o professor, assim, há uma relação mais livre entre monitor e estudante, pois, quando ocorre essa liberdade o estudante fica mais confortável para perguntar ao monitor já que, enquanto alunos, encontram-se no mesmo nível acadêmico, contribuindo, assim, para um maior aproveitamento. ${ }^{8}$

Fica notório que quando o estudante se torna monitor, este desenvolve atividades junto aos docentes e demais estudantes do curso fazendo com que o monitor tenha segurança no seu 
desempenho e estimula o estudante a desenvolver o trabalho em equipe, tendo assim melhor rendimento no âmbito universitário. ${ }^{12}$

Para que os estudantes se tornem monitores, é necessário dar a eles autonomia e responsabilidade para a construção das atividades, ou seja, é preciso estabelecer um vínculo de confiança entre o docente e o monitor. A confiança desperta o senso de responsabilidade dos monitores para com os seus colegas, e o sentimento que não podem decepcionar os professores. ${ }^{13}$

\section{Incentivo para estudar e momento de relembrar o que aprendeu}

Os depoimentos dos alunos revelam que a monitoria se configurou em uma estratégia para que buscassem relembrar o que já haviam aprendido e foi um incentivo para buscar novos conhecimentos, pois, quando não se sentiam seguros com algum tema ou procedimento buscavam aprender para repassar aos colegas. Estas afirmativas foram mencionadas nas seguintes falas:

Chance de estar relembrando as técnicas e a teoria e quando eu não sabia algo ia estudar. (M2)

Ser monitor foi demais para relembrar os procedimentos e sua relação com a teoria. (M4)

Recordei de muitas técnicas e sua associação com a teoria, pois, nem tudo eu havia praticado durante o estágio no hospital ou unidade básica. (M7)

Foi uma oportunidade de poder revisar e atualizar todos os procedimentos e a teoria que havia aprendido. (M8)

A monitoria forçou eu estudar, pois havia assuntos que eu não sabia mais. Então, fui estudar para aprender e repassar para os meus colegas. (M13)

A monitoria exige do aluno uma busca contínua pelo aprofundamento teórico e prático, fortalecendo seu aprendizado, o que irá gerar um sentimento de autoconfiança e responsabilidade, bem como estimula os estudantes de uma maneira geral, pois percebem que o monitor é um estudante assim como eles, que por seus esforços teve a capacidade de 
desenvolver um conhecimento avançado sobre um determinado assunto, e isso os deixa convictos de que também são capazes de atingir um bom nível de conhecimento. ${ }^{5-6}$

O monitor, para desenvolver suas atividades, busca atualização e aprofundamento científico para que possa desempenhar sua função com qualidade e, consequentemente, oferecer um maior aproveitamento dos estudantes. Evidencia-se que esse aperfeiçoamento deve ser contínuo, o que leva a concluir que essa experiência tem a capacidade de promover o aprendizado do monitor, tanto pela necessidade de revisar o que lhe foi ensinado, como a buscar de novos saberes, assim, a monitoria desenvolve no estudante um constante incentivo para buscar novos conhecimentos e atualizações. ${ }^{14}$

Denota-se que a monitoria provoca nos monitores um incentivo para estudar continuamente, pois é exigido conhecimento tanto pelo docente responsável pela monitoria que tem como função estimular a leituras para aprofundamento teórico-prático, mas também da necessidade do monitor que precisa levar aos colegas a discussão de diferentes temas, bem como a resolução de problemas que são evidenciados na prática clínica do enfermeiro. ${ }^{6-7}$

As atividades de monitoria possibilitam troca de experiência e proporcionam a solidificação de conhecimentos teórico-práticos e, principalmente, melhoram a relação com os docentes da disciplina e demais acadêmicos do curso de enfermagem. ${ }^{2}$

A monitoria é um processo de formação, pois há trocas de experiências, reforça seus conhecimentos e facilita os relacionamentos junto com os docentes e os discentes. ${ }^{1,4,10}$ Vale ressaltar que o monitor é a ponte entre o professor e estudante e tem como função principal revisar e fortalecer os conteúdos estudados em sala de aula. Entretanto, é preciso acompanhamento dos docentes continuadamente, pois o monitor também é um estudante e pode apresentar as mesmas dificuldades e ao buscar sanar suas dúvidas com o docente há um enriquecimento de conhecimentos entre todos os envolvidos. ${ }^{15}$ 


\section{Colaborando para a formação profissional}

Pode se observar pelas falas que ser monitor foi uma ferramenta importante para desenvolver a comunicação e para despertar sobre as responsabilidades que terão ao se tornarem profissionais.

Éramos estimulados a desenvolver nossa comunicação durante as monitorias, pois eu tinha que ensinar as técnicas e a fundamentação teórica. Assim, a comunicação era importante. (M10)

Aprendi a me comunicar melhor e tive uma visão da responsabilidade que teremos quando formos enfermeiras. (M3)

Os depoimentos mostraram que os alunos consideraram a monitoria como favorecedora da comunicação. Uma pesquisa teve resultados semelhantes ao do presente estudo, ao desvelar que a monitoria propiciou o desenvolvimento de habilidades de comunicação e que contribuirá para a prática profissional e o crescimento pessoal. ${ }^{16}$

Estudo $^{17}$ identificou que o programa de monitoria contribuiu para o entendimento do que é ser enfermeiro. A monitoria propicia a compreensão de como será o exercício profissional na prestação de cuidados, gestão e gerenciamento dos serviços de saúde e de enfermagem e no ensino, além de mostrar a importância da participação política e no controle social. A monitoria desperta para o desenvolvimento de uma postura ética e política diante da profissão, visto que propicia o desenvolvimento de habilidades e atitudes que são essenciais para a profissão. ${ }^{17}$

Como limitação do estudo indica-se a impossibilidade de generalizações dos resultados, considerando que os entrevistados foram apenas monitores de um curso de graduação em enfermagem. Entretanto, o estudo contribuiu para o avanço do conhecimento, visto que a monitoria se configurou em uma importante ferramenta para o ensino e aprendizado dos estudantes de enfermagem, mostrando aos gestores e docentes de cursos de graduação a relevância de implementar a monitoria em seus cenários. 


\section{Considerações finais}

Pode-se afirmar que a monitoria se constituiu como uma ferramenta importante para o desenvolvimento de habilidades teórico-práticas, que são importantes para o crescimento pessoal e profissional. Acredita-se que seja de suma importância a implantação de programas de monitorias acadêmicas nos cursos de graduação, considerando que auxiliam os monitores no despertar para a profissão da docência, no incentivo para o estudo, na ampliação e aprimoramento do conhecimento. Ainda, pode-se afirmar que é uma atividade que promove a interação entre os próprios estudantes e os docentes, além de contribuir na visualização e reflexão sobre o exercício profissional na área.

Portanto, as universidades devem investir e incentivar os programas de monitoria nas áreas que forem pertinentes. No que concerne à enfermagem é essencial, visto que a monitoria se configura em um recurso que possibilita um melhor preparo dos discentes para a prática em campo de estágio, bem como, o aprimoramento de técnicas, sendo que esse fator irá repercutir em uma melhor assistência de enfermagem aos pacientes e familiares.

Ressalta-se a importância de inserir novas práticas de ensino nas universidades, com destaque para a simulação realística com manequins de alta fidelidade que proporcionará uma monitoria mais próxima da realidade hospitalar.

Por fim, enfatiza-se a necessidade do desenvolvimento de novos estudos sobre essa temática, visto que foi desvelada a importância da monitoria acadêmica no processo de ensinoaprendizado do estudante do curso de enfermagem.

\section{Referências}

1. Bezerra MGM, Santos GA, Silva ME, Silva PS, Figueiredo NMA. O papel do monitor no curso de enfermagem do UNIFESO: relato de experiência a partir dos eixos de atuação pedagógica. Espaç Saúde. 2014;15(1):284-94. 
2. Baricati CCA, Martins JT, Yagi MCN, Kreling MCGD, Karino ME, Volpato MP. Monitoria: metodologia ativa na prática do cuidar em um curso de enfermagem. Braz J Surg Clin Res. 2017;21(1):769.

3. Santos GM, Batista SHSS. Monitoria acadêmica na formação em/para a saúde: desafios e possibilidades no âmbito de um currículo interprofissional em saúde. ABCS Health Sci. 2015;40(3):203-07.

4. Fernandes NC, Cunha RR, Brandão AF, Cunha LL, Barbosa PD, Silva CO et al. Monitoria acadêmica e o cuidado da pessoa com estomia: relato de experiência. Rev Min Enferm. 2015;19(2):238-41.

5. Gurgel SS, Taveira GP, Matias EO, Pinheiro PNC, Vieira NFC, Lima FET. Jogos educativos: recursos didáticos utilizados na monitoria de educação em saúde. Rev Min Enferm. 2017;21:e-1016.

6. Maran E, Tostes MFP, Melo WA, Spigolon DN, Teston EF. Habilidades e dificuldades técnicocientíficas dos acadêmicos de enfermagem durante a monitoria de semiotécnica. Rev Enferm UFPE on line. 2017;11(5):1819-25.

7. Frison LMB, Moraes MAC. As práticas de monitoria como possibilitadoras dos processos de autorregulação das aprendizagens discentes. Poíesis Pedagógica. 2010;8(2):144-58.

8. Silveira E, Sales F. A importância do programa de monitoria no ensino de biblioteconomia da Universidade do Estado de Santa Catarina (UDESC). InCID: Rev Cien Inf Doc. 2016;7(1):131-49.

9. Abreu TO, Spindola T, Pimentel MRAR, Xavier ML, Clos AC, Barros AS. A monitoria acadêmica na percepção dos graduandos de enfermagem. Rev Enferm UERJ. 2014;22(4):507-12.

10. Bardin L. Análise de conteúdo. São Paulo: Edições 70, 2016; 229 p.

11. Souza FMS, Barboza LC. A prática de monitoria no ensino de psicologia: ciência e profissão. Interbio. 2014;8(1):17-23.

12. Matoso, LML. A importância da monitoria na formação acadêmica do monitor: um relato de experiência. Rev Cient Esc Saúde. 2014; 3(2):77-83.

13. Cunha Junior, FR. Atividades de monitoria: uma possibilidade para o desenvolvimento da sala de aula. Educ. Pesqui. 2017. 43(3):681-694.

14. Vicenzi CB, Conto F, Flores ME, Rovani G, Ferraz SCC, Marostega MG. A monitoria e seu papel no desenvolvimento da formação acadêmica. Rev Ciênc Ext. 2016;12(3):88-94.

15. Santos M, Batista SHSS. Monitoria acadêmica na formação em/para a saúde: desafios e possibilidades no âmbito de um currículo interprofissional em saúde. ABCS Health Sci. 2015;40(3):203-07. 
16. Andrade EGR, Rodrigues ILA, Nogueira LMV, Souza DF. Contribuição da monitoria acadêmica para o processo ensino-aprendizagem na graduação em enfermagem. Rev Bras Enferm. 2018;71(Suppl 4):1596603.

17. Andrade SR, Piccoli T, Ruoff AB, Ribeiro JC, Sousa FM. Fundamentos normativos para a prática do cuidado realizado pela enfermagem brasileira. Rev Bras Enferm. 2016;69(6):1082-90.

\section{Autor correspondente}

Alessandro Rolim Scholze

E-mail: scholze@uenp.edu.br

Endereço: Rua: Prefeito José Mario Junqueira, 393 ap. 01 Bandeirantes - Paraná, CEP: 86.360-000

\section{Contribuições de Autoria}

1 - Cinthya das Neves Burgos

Concepção e planejamento do projeto de pesquisa, obtenção dos dados.

2 - Crysthianne Cônsolo de Almeida Baricati

Concepção e planejamento do projeto de pesquisa, análise e interpretação dos dados, redação e revisão crítica.

3 - Júlia Trevisan Martin

Concepção e planejamento do projeto de pesquisa, análise e interpretação dos dados, redação e revisão crítica.

4 - Alessandro Rolim Scholze

Concepção e planejamento do projeto de pesquisa, análise e interpretação dos dados, redação e revisão crítica.

5 - Maria José Quina Galdino

Concepção e planejamento do projeto de pesquisa, análise e interpretação dos dados, redação e revisão crítica.

6 - Marcia Eiko Karino

Concepção e planejamento do projeto de pesquisa, análise e interpretação dos dados, redação e revisão crítica. 
Monitoria acadêmica na percepção dos estudantes de enfermagem | 14

\section{Como citar este artigo}

Burgos CN, Baricati CCA, Martins JT, Scholze AR, Galdino MJQ, Karino ME. Monitoria acadêmica na percepção dos estudantes de enfermagem. Rev. Enferm. UFSM. 2019 [Acesso em: 2019 jun 15];vol 9 ex:114. DOI:https://doi.org/10.5902/2179769230816 